\title{
Study on the Model of Quality Safety Information Diffusion
}

\author{
Wuyi Zhang $1^{\text {st }}$ \\ Quality Development Institute \\ Kunming University of Science and Technology \\ Kunming, China \\ e-mail: wuyi20000@yahoo.com.cn \\ Yunfei Yang $2^{\text {nd }}$ \\ Faculty of Management and Economics \\ Kunming University of Science and Technology
}

Abstract-Based on the SI communicable disease model, this paper established the model of quality safety information diffusion by comparing the system composition of SI model and the diffusing process with the model of quality safety information diffusion. Then, the paper overcomes the defect that $\alpha$ and $\beta$ are fixed value in SI model and makes a piecewise function, which changes with the independent variable $\tau$. By using MATLAB, we emulate quality safety information diffusion process with a non-network and full

\section{INTRODUCTION}

Quality safety information is intrinsic quality information of the product or service. Under normal circumstances, it is disseminated by the general channels, such as $3 \mathrm{C}$ safety certification, product quality meets, etc. And the quality information poses a real or potential threat to consumer's safety, such as life safety, physical safety and health. Sanlu incident in 2008 and liquor plasticizer incident in 2012 are both typical 1 explosive dissemination events of quality and safety information. In the process of safety quality information diffusion, the people would suspect the correct quality information and refuse the similar products or services because of panic and then the industry would shrink back. From the downturn of the Chinese dairy industry to 400 billion evaporated in listed companies of Chinese liquor industry' market during a month, all these show that both

\author{
Kunming, China \\ e-mail: 845735130@qq.com \\ Linjing Tao ${ }^{3 \mathrm{rd}}$ \\ Quality Development Institute \\ Kunming University of Science and \\ Technology \\ Kunming, China \\ 269529688@qq.com
}

network combination pattern as well as different public attention, finding some regularities of quality safety information diffusion. Finally, the whole process of hazards information diffusion and the peak time $t$ of diffusion are forecasted in a case study of Yunnan rice noodle. Therefore, decision making departments could take measures before the peak time to avoid social panic.

Keywords- infectious disease model; quality safety; information diffusing model

enterprises and related government didn't diffuse safe quality information under the operating regularities. Not only it caused irreparable damage on the brands, but also more of the industries have a huge blow. The diffusion of quality and safety information is the spreading process from the outbreak to the peak and then the recession. By grasping information diffusing regularities of the quality and safety and taking appropriate preventive measures, it could effectively guide consumers to obtain the correct information and avoid social panic.

SI model is the infectious disease model and some studies have been done based on it, such as knowledge diffusion ${ }^{[1]}$, the process of study atmosphere $^{[2]}$. So far, no scholar has done any quantitative analysis to the quality safety information diffusing process. Based on the similarity between the SI model system structure and the process of quality and safety information diffusing model, this paper builds up quality and 
safety information diffusing model and simulates diffusing process by MATLAB and provides new measures and ways for diffusing study.

\section{II.THE SIMILARITY BETWEEN THE DIFFUSION OF SAFETY QUALITY INFORMATION AND THE TRANSMISSION OF INFECTIOUS DISEASES}

\section{A. Infectiousness}

The excreted infectious "pathogens" will infect the new vulnerable people in a certain way. When people obtain and understand the quality safety information, they will spread the information to information-unknown people by contacts or Internet, which shows the feature of infectiousness. And the transmission intensity is related to interpersonal relations, networking influences and other factors.

\section{B. Epidemicity}

If no prevention or any control measures of epidemic diseases are taken, there will be severe consequences within a certain period of time. The quality safety information diffusion has the epidemicity. In a certain area or a certain period of time, once the information appears, it will spread rapidly and then cause panic or even more serious consequences.

\section{Re-infectiousness}

Patients who have recovered from some infectious diseases like colds, diarrhea or other disease have low immunity. We can assume these people are immunity-free. So patients could recover, while the healthy could get re-infected. In the process of the information diffusion, some people turn into information spreaders from information unknowns. If another quality safety information event breaks out, they will become spreaders again.

As can be seen from the above, the diffusion of quality safety information has much in common with the spreading process of infectious diseases. Therefore, it is feasible to investigate the diffusion of quality safety information based on the spreading process of infectious disease.

\section{MODELING}

\section{A. Model assumption}

(1) Taking no consideration of group dynamics factors such as birth, death and migration, that is, making the number of total population $\mathrm{N}(\tau)$ to be fixed. And its value remains at a constant $\mathrm{N}$.

(2) Assuming that the population is divided into two groups: $\sigma$ (Information unknowns), $\imath$ (Information spreaders). $\sigma(\tau)$ is the proportion of those information unknowns in the total population, which means those people are still not influenced, but will potentially accept some information at time $\tau . \imath(\tau)$ is the proportion of spreaders in the total population, which means the people who know the safe quality information and will diffuse them.

(3) $\alpha$ is diffusion rate, which indicates the number of population every spreader diffuses to.

(4) $\beta$ is exit rate, which means the proportion of spreaders who will exit.

$$
\begin{aligned}
& \text { (5) Assuming the initial state is: } \\
& \sigma(0)=\sigma_{0}, \imath(0)=\mathfrak{l}_{0} \text {. }
\end{aligned}
$$

\section{B. Model derivation}

Based on the assumption, every spreader diffuses the information to $\alpha \sigma(\tau)$ information unknowns. Because the number of spreaders is $\mathrm{Nl}(\tau)$, so there are $\alpha \sigma(\tau) \mathrm{N}_{\imath}(\tau)$, information unknowns become spreaders. But there will be $\beta \mathrm{N}$ i $(\tau)$ spreaders exit every day.

So the spreader model is:
$\mathrm{N} \delta \mathrm{l} / \delta \tau=\alpha \sigma(\tau) \mathrm{Ni}(\tau)-\beta \mathrm{Nl}(\tau)$
Simplify the equation
$\delta \mathrm{l} / \delta \tau=\alpha \sigma(\tau) \mathrm{t}(\tau)-\beta \mathrm{t}(\tau)$

The increasing number of information unknowns can be expressed as:

$$
\mathrm{N} \delta \sigma / \delta \tau=-\alpha \sigma(\tau) \mathrm{Ni}(\tau)
$$

In the model, the minus sign refers to the direction of diffusion. By simplifying the equation (3), the increasing rate is obtained as:

So the quality safety information diffusion model is: 
$\delta \sigma / \delta \tau=-\alpha \sigma(\tau) \imath(\tau)$

So the quality safety information diffusion model is:

$$
\left\{\begin{array}{l}
\delta \mathrm{l} / \delta \tau=\alpha \sigma(\tau) \mathfrak{l}(\tau)-\beta \mathrm{t}(\tau) \\
\delta \sigma / \delta \tau=-\sigma(\tau) \mathfrak{\imath}(\tau) \\
\sigma(0)=\sigma_{0} \\
\imath(0)=\mathfrak{l}_{0} \\
\sigma(0)+\mathfrak{\imath}(0)=1
\end{array}\right.
$$

With the help of software MATLAB, this paper simulates the diffusion process of the quality and safety information through programming language.

IV. The simulation on process of quality and safety information diffusion

The assumption that $\alpha, \beta$ have fixed values in SI model would ignore the current situation that the number of diffusing population and exiting population change with the time. So there will lead to errors between simulated diffusion process and the reality of the situation. In order to make simulation diffusion process and the reality more suitable, this paper overcomes the defect of SI model and makes it better by taking $\alpha, \beta$ as dynamic constants changing with the time factor $\tau$ and establishes a piecewise function.

This paper divides the process of safe quality diffusing information into two categories. The first simulating model depends on the combination mode of non-network and full network. The second simulating model depends on different public attention. Following part will set up a section function depending on the two categories in real situations. The $\alpha, \beta$ piecewise function changes with $\tau$.

A. Simulating the process of quality safety diffusing information under different modes

In reality, the diffusing process of quality safety information is a combination of non-network with network.

Assuming the initial value is :

$\imath(0)=0.000000005, \sigma(0)=0.999999995$

The $\alpha, \beta$ piecewise function changes with $\tau$ :

$$
\alpha=\left\{\begin{array}{cc}
1.3 & 0 \leqslant \tau<30 \\
2 & 30 \leqslant \tau<45 \\
0.8 & 45 \leqslant \tau<60
\end{array} \quad \beta=\left\{\begin{array}{cc}
0.550 \leqslant \tau<30 \\
0.4530 \leqslant \tau<45 \\
0.2545 \tau<60
\end{array}\right.\right.
$$

Under a combination of non-network with network situation, safe quality diffusing information is simulated by MATLAB. The result is shown in Figure 1:

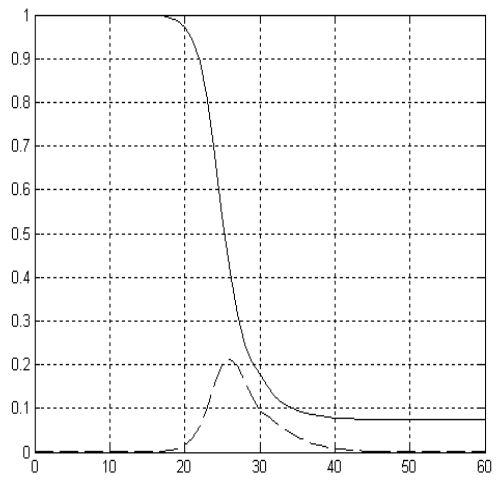

Figure1. $\sigma(\tau)$ and $\imath(\tau)$ change with time $\tau$

Note: the full line shows $\sigma(\tau)$, the dotted line shows $\mathrm{l}(\tau)$, the horizonta axis: days, the vertical axis: proportion. The same is true of the figures below.

From Figure 1 we can know: $\sigma(\tau)$ monotonically decrease, eventually tends to a small value as $\tau \rightarrow \infty . \quad l(\tau)$ monotonically increase at first, then monotonically decrease after the peak, finally approaches zero with increasing $\tau$.

B. Simulating the diffusing process of quality safety information with different public attentions

The 2010 Survey of the senses of security in China includes: social security safety, food safety, traffic safety, environmental security and so on. Results of the survey show that public concern of food quality safety is the highest with $72 \%$. The attention to quality of medical care takes up 55\%, and environmental quality safety reaches at $39 \%{ }^{[3]}$. According to different attentions and specific cases, the three modes of process have been simulated by MATLAB.

(1) Diffusing process of food quality safety information

The quality safety issue with great concerns is food quality safety. Taking the events of 
shuanghui for example, assume that: 10 persons know this incident at first. This incident is a national event, because shine way products are sold nationwide. China has a population of 1.3 billion, so the proportion of the number of initially-known people in the total population is $\imath(0)=0.000000008$, model (5) shows: $\sigma(0)$ $=0.999999992$.

The $\alpha$ and $\beta$ piecewise function is changing with t:

$\alpha=\left\{\begin{array}{cc}2 & 0 \leqslant \tau<20 \\ 4 & 20 \leqslant \tau<40 \\ 1.5 & 40 \leqslant \tau<60\end{array} \quad \beta=\left\{\begin{array}{cc}0.55 & 0 \leqslant \tau<20 \\ 0.45 & 20 \leqslant \tau<40 \\ 0.25 & 40 \leqslant \tau<60\end{array}\right.\right.$

Food quality safety information diffusion is simulated by MATLAB. The result is shown in Figure2:

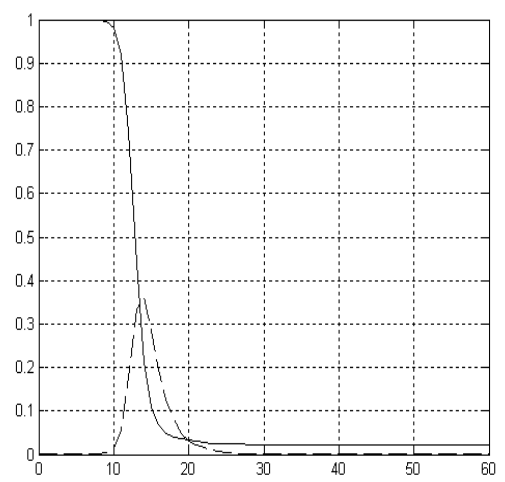

Figure2. $\sigma(\tau)$ and $\imath(\tau)$ change with time $\tau$

(2) Diffusing process of medical care quality safety information

Medical care incidents are local events, whose impacts are limited in region. Assume that the value of $\mathrm{N}$ is the population of an area. In order to make assumptions more in line with the actual, this paper takes a medical negligence in Kunming for example. Fan Tianying used the First Affiliated Hospital of Kunming Medical College ${ }^{[4]}$. Assuming 5 persons know this incident at first, the population of Kunming is 6.43 million. So the proportion of the number of initial known person in the total population is: $\imath(0)=0.000000778$. Model (5) shows: $\sigma(0)$ $=0.999999222$.

The $\alpha$ and $\beta$ piecewise function is changing with $\tau$ :

$\alpha=\left\{\begin{array}{lc}1.3 & 0 \leqslant \tau<25 \\ 2 & 25 \leqslant \tau<40 \\ 0.8 & 40 \leqslant \tau<60\end{array} \quad \beta=\left\{\begin{array}{cc}0.56 & 0 \leqslant \tau<25 \\ 0.46 & 25 \leqslant \tau<40 \\ 0.26 & 40 \leqslant \tau<60\end{array}\right.\right.$

Quality of medical care diffusing information is simulated by MATLAB. The result is shown inFigure3:

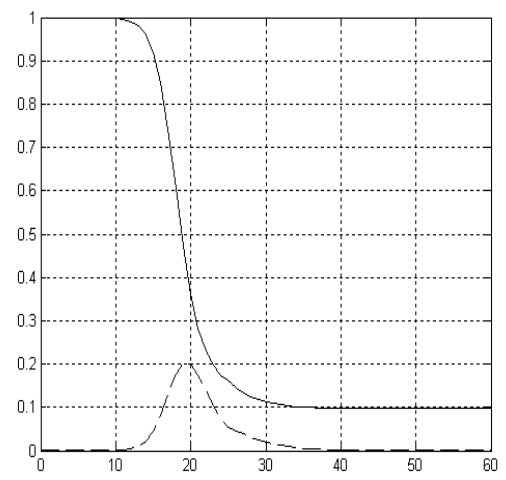

Figure3. $\sigma(\tau)$ and $\imath(\tau)$ change with time $\tau$

(3) Diffusing process of environmental quality safety information

Environmental security issue is of less public concerns. These incidents belong to local events. The pollution of double benzene by Petro China of Jilin Province in 2005 is taken for example ${ }^{[5]}$. Assuming: 4 persons know this incident at first. The population of Jilin City is 4.51 million. So the proportion of the number of initial known person in the total population is: $\imath(0)=0.000000887$, model $(5)$ shows: $\sigma(0)$ $=0.999999113$.

The $\alpha$ and $\beta$ piecewise function is changing with $\mathrm{t}$ :

$\alpha=\left\{\begin{array}{ll}1.1 & 0 \leqslant \tau<30 \\ 1.5 & 30 \leqslant \tau<45 \\ 0.5 & 45 \leqslant \tau<60\end{array} \quad \beta=\left\{\begin{array}{cc}0.57 & 0 \leqslant \tau<30 \\ 0.47 & 30 \leqslant \tau<45 \\ 0.27 & 45 \tau \leqslant<60\end{array}\right.\right.$

Environmental quality safety diffusing information is simulated by MATLAB. The result is shown in Figure4: 


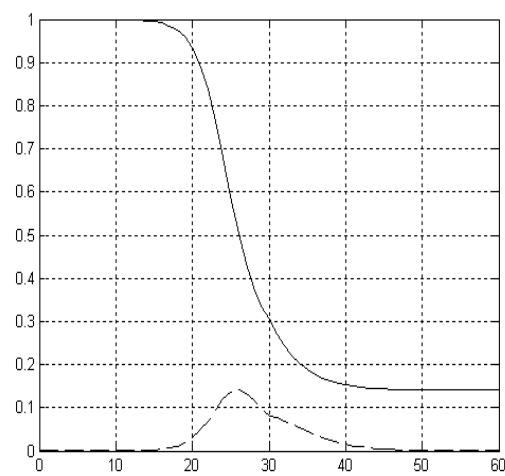

Figure4. $\sigma(\tau)$ and $\imath(\tau)$ change with time $\tau$

From Figure2 Figure3 and Figure4, we can conclude that: (1) the changing rules of $\sigma(\tau)$ and $\imath(\tau)$ are the same. They change with $\tau(2)$ there is a positive correlation between public attention and information diffusing speed. From the three examples: the ratio of public attention to food quality safety is in the highest and the $t(\tau)$ takes the shortest time to reach the peak. Which means that food quality safety information diffuse fast. The ratio of public attention to environmental security is the lowest. The $\imath(\tau)$ takes the longest time to reach the peak. Which means that environmental security information diffuse slowest. The speed of medical care quality is between food quality safety and environmental security. So public attention degree to quality safety information affects the diffusing speed, and they have a positive correlation.

\section{V.FORECAST THE DIFFUING PROCESS OF QUALITY SAFETY \\ INFORMATION-TAKING THE PROCESS OF FOOD FOR EXAMPLE}

Depending on modes of different public attention degrees, this paper has simulated the process of quality safety diffusing information by MATLAB. The results show the diffusing regularities of safe quality information. So we take the founded regularities as a technical support and base on food safety hazard in real life, as well as forecast how quality safety information of food diffuses. And calculates when the diffusing number reaches the peak, which means the time $t(\tau)$ reaches to. If relevant government departments take restraining measures before diffusing peak, the social panic could be avoided.

Rice noodle is prestigious in Yunnan province, especially in Kunming. But maybe there exists food quality safety hazard in some rice noodle producers or restaurants. They are pork parasite infection, bleaching powder additives and so on. Therefore, if relevant government departments take no restraining measures, some merchants have weak awareness of food quality and safety and quality unsafe incidents will occur in the future. Assuming that some producers add large doses of alum, rice noodle quality unsafe incidents occur. Assuming: 10 persons know this incident at first. The population of Kunming is 6.43 million. So the proportion of the number of initial known person in the total population is:

$\mathrm{l}(0)=0.000001555$, model (5) shows: $\sigma(0)$ $=0.999998445$.

The $\alpha, \beta$ piecewise function changes with $\mathrm{t}$ :

$\alpha=\left\{\begin{array}{ll}2.1 & 0 \leqslant \tau<15 \\ 4.5 & 15 \leqslant \tau<35 \\ 1.8 & 35 \leqslant \tau<60\end{array} \quad \beta=\left\{\begin{array}{lc}0.53 & 0 \leqslant \tau<15 \\ 0.43 & 15 \leqslant \tau<35 \\ 0.23 & 35 \leqslant \tau<60\end{array}\right.\right.$

The process of diffusing information is simulated by MATLAB. The result is shown in Figure5:

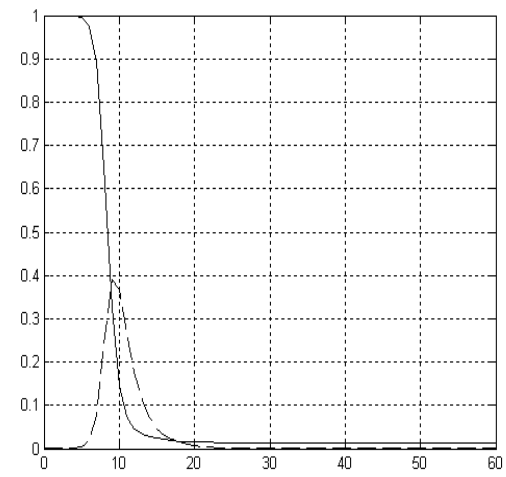

Figure5: $\sigma(\tau)$ and $\mathrm{t}(\tau)$ change with time $\tau$

Considering the whole process of diffusion, if quality unsafe incident of rice noodle occurs, the proportion reaches the maximum when the time is $\tau=9$. Which means diffusion reaches to the peak 9 days later. So in order to avoid social 
panic, relevant government departments should

\section{SUMMARY}

This paper has set up a quality and safety diffusing information model based on SI model. The feasibility also has been proved by the similarity of SI model and model of quality safety diffusing information. This paper simulated the process of quality safety diffusing information. The diffusing regularities have been found at the same time. Finally, taking the founded diffusing regularities as a technical support, the process of food quality safety incident could be forecasted. Therefore, relevant government departments could take restraining take restraining measures before the peak time. measures to avoid social panic before diffusing peak time when quality safety incident occurs. The value $\alpha$ and $\beta$ would have changed as soon as the time $\tau$ had changed. In this paper, the defect of fixed value of SI model has been overcome. According to actual situation, this model takes $\alpha$ and $\beta$ as dynamic constants changing with the time factor $t$ and sets up a piecewise function. So it can simulate the process of quality safety diffusing information. Assumed $\alpha$ and $\beta$ values are biased between the assuming and the accurate. But the model will be enriched and deepened in the following studies.

\section{ACKNOWLEDGEMENT}

Fund Project: Reserve talents fund for young and middle-aged academic technology leader in Yunnan Province; Fund Code: 2010CI010.

\section{REFERENCES}

[1] LiuYuan. Epidemic model used in tatic knowledge diffusion $[\mathrm{J}]$. Science \& Technology Progress and Policy. 2008, 25(7): 168-171.

[2] XueLing. Learning environment research based on epidemic model [J]. Grand View Weekly.2012, 587(29): 256-258.

[3] People worry about food safety problems most, often overhalf[EB/OL].http://news.sina.com.cn/c/sd/2010-06 -30/111620579550.shtml,2010-06-30.
[4] Tianying Fan, Yufen Fan litigates First Affiliated Hospital of Kunming Medical College on medical malpractice[EB/OL].http://www.docin.com/p-3709770 34.html, 2004.

[5] The province's public emergencies assessment analysis in 2005[EB/OL].http://www.hlj.gov.cn/yjgl/system/200 7/10/10/000072200.shtml, 2006-04-05. 